\title{
Suburbanization Effect of Urban outward Expansion in the New-type Urbanization and the Competitive Strategy of Assembled Building Developers
}

\author{
Gangqiang Luo ${ }^{1 *}$ and Qin Wang ${ }^{1}$ \\ ${ }^{1}$ Department of Civil and Construction Engineering, Guangdong institute of Arts and Sciences, Zhanjiang, Guangdong, 524400, China \\ ${ }^{1}$ Department of Finance and Accounting, Guangdong institute of Arts and Sciences, Zhanjiang, Guangdong, 524400, China
}

\begin{abstract}
This paper expounds the meaning of urban suburbanization in a narrow sense and broad sense, and probes into the dynamic mechanism of suburbanization. The influence of the suburbanization effect of the urban outward expansion on the assembled building developers is analysed from three aspects: the selection of the target market, the positioning of the market and the assembled building development structure. Based on the Salop Circumferential City Model, two stages of game analysis are carried out for the competition of assembled building developers. The research results show that in the process of suburbanization of most cities in China at present, it still stays at the stage of circular competition. With the continuous agglomeration of large population, industry and commerce in the suburbs of cities, the circular competition pattern of developers will be transformed into plate competition pattern.
\end{abstract}

\section{Introduction}

From the perspective of the supply of developers, the rapid development of urbanization makes the construction land resources of urban central area extremely limited, and the competition among assembled building developers will become increasingly fierce ${ }^{[1]}$. The strong crowding out effect of urban plate competition will lead some assembled building developers to turn to suburban areas for development, thus making the potential advantages of urban suburban areas gradually become the focus of assembled building developers' strategic development goals ${ }^{[2]}$. From the perspective of buyers demand, with the accelerating pace of infrastructure construction in rural areas, the increasingly convenient development of urban public transport, light rail transit, subway and network communications, and the continuous shift of the focus of modern urban living and living space, more and more buyers are attracting suburban suburbs ${ }^{[3]}$.

\section{Suburbanization effect of urban outward expansion for assembled building development}

\subsection{The narrow sense and broad sense meanings of suburbanization}

Suburbanization is a centrifugal diffusion phenomenon of urban agglomeration to a certain stage, which leads to the development of the suburbs ${ }^{[4]}$. However, the improvement of residents' income level and the improvement of transport infrastructure have promoted the suburbanization of China. The reduction of traffic cost and the increase of income level will trigger the outward migration of urban population, which will lead to the expansion of urban boundaries. With the migration of urban middle and upper class population to urban suburban areas, the urbanization process in suburban rural areas outside the central urban area is called suburbanization. The result of the reduction of traffic cost and the increase of income level is the result of suburbanization, which is called suburbanization power (as shown in Figure 1). 


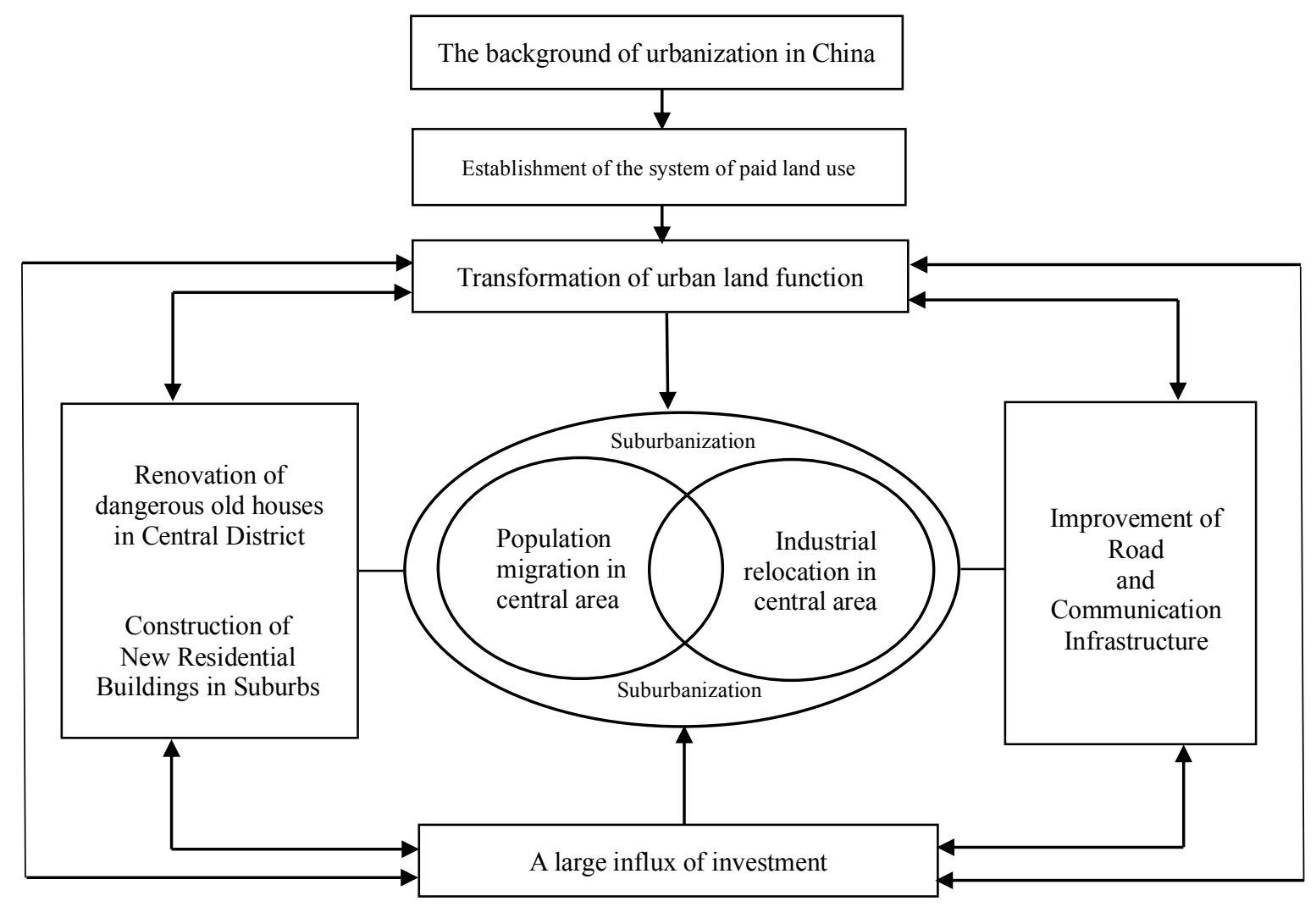

Figure 1. Dynamic mechanism of suburbanization under the background of new-type urbanization in China

Suburbanization refers to the process of migration of population, industry and employment from the center of a big city to the suburbs. In the West, the urban center is the administrative central city, and the suburbs are built areas or metropolitan areas outside the central city ${ }^{[5]}$. In some big cities of China, there are "circle-type" regional division, with relatively clear and consistent concepts of central areas with administrative boundaries, such as Beijing, etc (as shown in Figure 2).

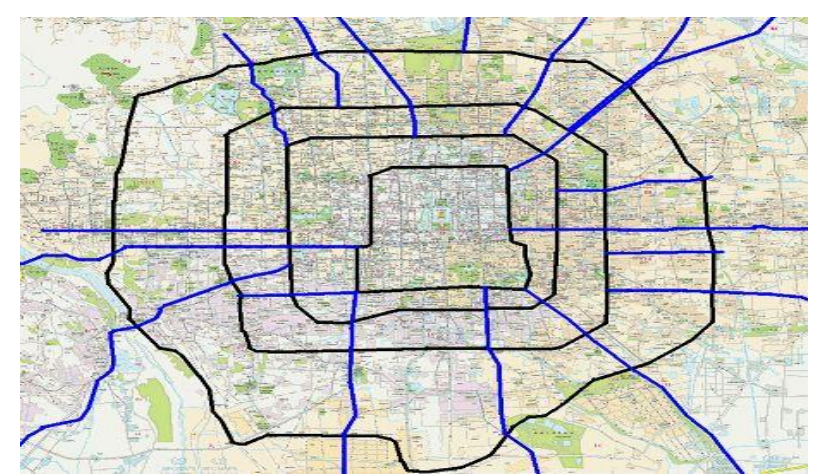

Figure 2. Beijing's concentric circular structure and suburbanization of urban outward expansion

While some cities are "partitioned" regional division, without the existing concept of central areas, researchers need to refer to the present according to the historical process of urban development. In order to determine the land grade and population density, it is sometimes necessary to break the existing administrative boundaries and take subdistrict offices as basic units, such as Shenyang, etc (as shown in Figure 3).

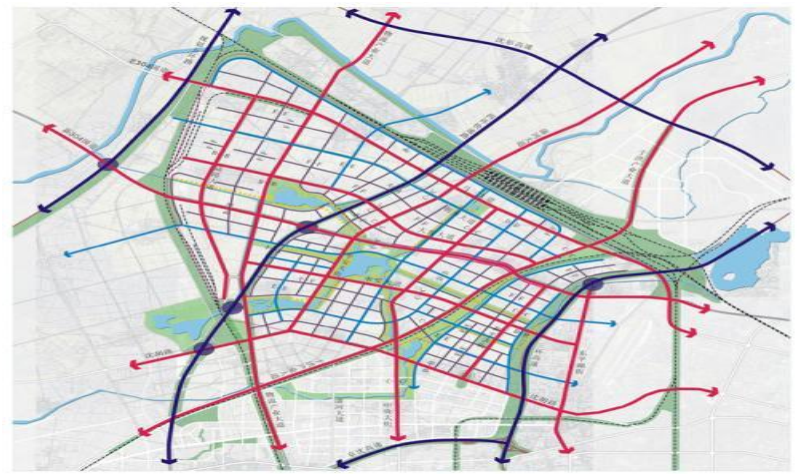

Figure 3. Shenyang's divided-type regional state and suburbanization of urban outward expansion

Although the suburbs of cities vary greatly, the suburbs of China generally refer to a part of the city that is divided and recognized by the state administration. It is inseparable from the city center in politics, economy, culture and other aspects, and developers together [6]. Despite the difference between suburbs, there are two main situations: one is the suburbs and counties under the jurisdiction of the city government; the other is the junction of cities.

There are different understandings about suburbanization in domestic and foreign theoretical circles. Generally speaking, there are two kinds of suburbanization: broad sense suburbanization and narrow sense suburbanization. Generalized suburbanizers believe that as long as the population (and functions) of the urban center migrate to the suburbs, suburbanization does not involve the stagnation and decline of urban area. The narrow sense of suburbanization holds that only the 
suburbanization caused by the population and function migration of the central city, and this suburbanization leads to the stagnation and decline of the central city, is the strict sense of suburbanization.

\subsection{Suburbanization effect of urban outward expansion for assembled building development: target market, location selection, and development structure}

\subsubsection{The realistic process of suburbanization affects the target market of assembled building development.}

The development of suburban assembled building should first understand whether urban development is centrally concentrated, centrifugal diffused or coexist. If it is centripetal concentration stage, the target market of suburban assembled building development should be mainly non-agricultural population in the suburbs, supplemented by the ordinary middle class working in the urban area to find cheap assembled building, developing middle and low-grade assembled building. If it is centrifugal diffusion, the target market of suburban assembled building development should be positioned in the urban middle class and the rich class, developing high-grade assembled building and villas. In the coexistence stage, the target market of suburban assembled building should be subdivided according to the characteristics of the plot ${ }^{[7]}$. There is no recession in the process of suburbanization in China. The central area of the city is more prosperous, and has more and more attraction to the economic activities such as population and commerce. This centrifugal diffusion and centripetal agglomeration coexist and the urban development stage based on agglomeration determines that the suburban assembled building development in China should be subdivided according to the specific characteristics of the suburban blocks, and the main development is low and medium development. High-grade assembled building can meet the housing needs of non-farmer population in suburbs and salaried class in urban areas, and develop high-grade assembled building and villas for the rich class in urban areas.

\subsubsection{The spatial expansion direction of suburbanization affects the location selection of suburb assembled building development.}

The development of suburban assembled building should be consistent with the direction of suburbanization, and make full use of the location advantages of traffic axes to develop suburban group assembled buildings. In the process of suburbanization, the population of urban area moves out along the main traffic routes and buys houses in the areas with relatively perfect supporting facilities, and the industrial enterprises in urban area concentrate further along the main traffic routes to the suburban development zones ${ }^{[8]}$. Therefore, the development of suburban assembled buildings and industrial factories can depend on the traffic axis and choose the suburban development zones and key towns for assembled building development. At present, most of the population in China's suburbanization is the ordinary working class who wants to increase the residential area. The trip mainly depends on the public transport system. The distance from population migration is not far away, and most of them are concentrated in the urban suburban area [9]. Accordingly, suburban assembled building development can choose ordinary commercial housing with relatively low construction price in the suburbs. The wealthy stratum in the urban area pursue more beautiful living environment, design and perfect supporting facilities. They generally own private cars and have a strong bearing capacity for transportation costs. High-end apartments and villas in the suburbs can be developed in areas with convenient transportation and far away from the urban area.

\subsubsection{The residential demand of migrants in suburbanization affects the development structure of suburb assembled buildings.}

People are interested in suburban residential consumption, which can be divided into four types: the first type is residential cultural consumption, which is interested in suburban environment, landscape and leisure mode; the second type is suburban vacation type, which can be used as a way of life promotion and supplement; the third type is residential utility type, which is interested in lower prices in suburban areas; the fourth type is assembled building investment type, which is optimistic about the assembled building development trend of suburban areas ${ }^{[10]}$. Population residence usually has the tendency of similar population aggregation, that is, people of similar cultural background or similar income level tend to live together, but in reality there are differences in occupations, income, social status and life concept, which leads to different assembled housing preferences. The residential consumption type of the ordinary wage earners in suburbanization belongs to the residential practical type ${ }^{[11]}$. The middle class usually belongs to the residential cultural type, while the rich class prefers the vacation leisure or investment utility of the residential. Therefore, the development structure of suburban assembled housing should be in line with the diversified living needs of the migrant population in suburbanization. With different types of population gathering in different suburban areas, the differentiation of social strata in space is manifested as the differentiation of suburban assembled housing.

\section{Ring competition strategy assembled building of developers based on the Salop Circumferential City Model ${ }^{[12]}$}

Many papers analyzed the competitive behavior of duopoly developers in the regional plate market by using Hotelling's "linear city" model. Due to suburbanization, the surrounding area of the developers is similar to the "ring city". Assuming that the suburbanization competition of the developers is in addition to fixed costs 
or entry costs, there are no other entry barriers and location choices and market pricing problems under the "entry barriers" conditions. Therefore, the circumferential city model can be introduced to the developers in the suburbs.

\subsection{The basic assumption of the Salop Circumferential City Model}

Suppose that consumers are not evenly distributed in online cities, but evenly distributed in the circumference of 1 , similar to "circumferential city". Businesses are also situated around the circle, where consumers search for their products. Consumers buy only one unit product at a time. The commuting cost per unit distance is $t$ (replaced by linear distance commuting cost for simplification), the transportation cost provided by enterprises is $c$, and the fixed cost is $f$. There are no barriers to entry except for the fixed cost that needs to be paid. It is assumed that there are $n$ firms entering the market, and any one of them is priced as $i$, and the demand it faces is $D_{i}$.

\subsection{Two-stage game analysis of ring competition among assembled building developers}

In the first stage, if the developer enters the suburbs at the same time, how to choose the location; in the second stage, if the location is selected, how to price the developer.

Game in the first stage: Potential assembled building developers decide whether to enter or not to indicate the number of developers entering. The idea of each developer in the game is to stay away from the adjacent competitors to strengthen their control over the surrounding market. The result of the competition is that the entry developer's choice of location is distributed equidistantly around the circumference, and the adjacent is $1 / \mathrm{n}$ of equidistant length (as shown in Figure 4).

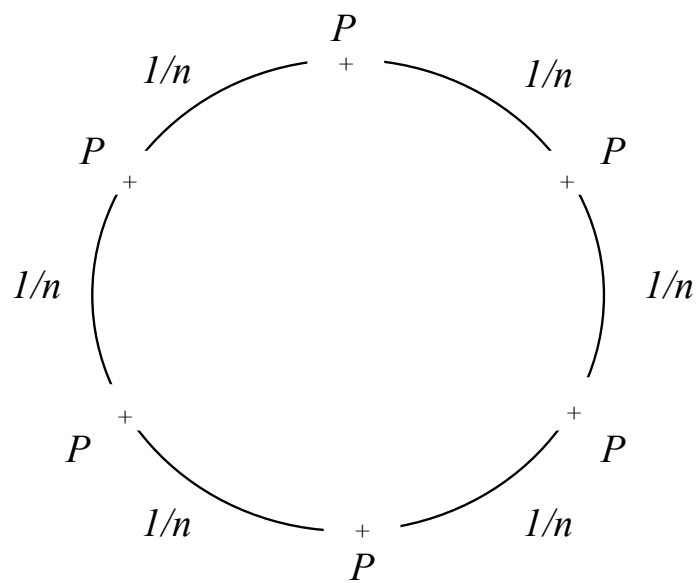

Figure 4. Salop Circumferential City Model and isometric distribution of suburban developers
If the house price given by developer $i$ is $p_{i}$ (as shown in Figure 5), when the distance between the buyer and the developer $i$ is $x \in(0,1 / n)$ and the condition $p_{i}+t x=p+t(1 / n-x)$ is satisfied, there is no difference for the buyer whether it is in the developer $i$ or in any of the adjacent developers $(i-1, i+1)$.

$$
P_{i}
$$

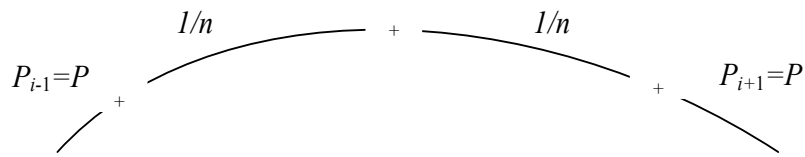

Figure 5. Ring competition between assembled building developers

Therefore, the demand function for house purchase faced by assembled building developers is as follows:

$$
D_{i}\left(p_{i}, p\right)=2 x=\left(p+t / n-p_{i}\right) / t
$$

The profit function of the assembled building developer $i$ is as follows:

$\pi_{i}=\left(p_{i}-c\right) D_{i}\left(p_{i}, p\right)-f=\left(p_{i}-c\right)\left(\frac{p+t / n-p_{i}}{t}\right)-f$

According to the first-order condition of maximizing revenue, under the assumption of $p_{i}=p$, the derivative of $p_{i}$ is obtained:

$$
p=c+\frac{t}{n}
$$

From the results of formula 3 , we can see that the price demanded by assembled building developers increases with the increase of commuting costs and decreases with the increase of the number of developers entering the market. Assuming that in the absence of any market entry barriers, the number and price of assembled building developers can be deduced based on the zero profit of developers.

$$
\left\{\begin{array}{l}
(p-c) \frac{1}{n}-f=\frac{t}{n^{2}}-f=0 \\
n^{*}=\sqrt{t / f} \\
p^{*}=c+\sqrt{t f}
\end{array}\right.
$$

From the above results, we can see that when the market reaches equilibrium, even if the price of the assembled building developer is higher than the marginal cost, it is still not profitable. The increase in fixed costs leads to a decrease in the number of assembled building developers and an increase in cost additions. Increased commuting costs increase the cost bonus and increase the number of developers. When there is no barrier to entry and fixed cost tends to zero, the number of assembled building developers entering the market will tend to be infinite, thus housing prices tend to marginal cost. Therefore, fixed cost constitutes a barrier to entry of the housing market in a certain sense, and its level has a great influence on the number of developers entering the market and the size of their profits. 


\section{Conclusion}

The practical significance of the circular competition of the assembled building developers in the suburbs of the Salop model lies in that, in the process of the developers entering the suburban market, under the effect of suburbanization, the competition among the developers distributed in the suburban "circular market" is mainly the circular competition of the adjacent developers, and the more distant the developers are, the less competitive they are. Under the relaxed assumption, if the housing products between developers have certain substitutability, there will be a limited number of assembled building developers competing in specific suburban areas, which will form overlapping market areas, but more developers have their own monopoly areas. Therefore, the bigger the monopoly area is, the weaker the competition among developers. When the overlapping market area is bigger and the overlapping market area is close to zero, the circular competition will turn into complete competition, which is a situation that developers are very unwilling to happen. At present, in the process of suburbanization of most cities in China, it still stays at the stage of circular competition. With the continuous agglomeration of large population, industry and Commerce in the suburbs of cities, the circular competition pattern of developers will be transformed into plate competition pattern.

\section{Acknowledgments}

This research project was supported by the Science and Technology Tackling Project of Zhanjiang, Innovation and Strong School Project funding of Guangdong institute of Arts and Sciences and the Special Funds for Education Development at Guangdong Provincial Level in 2019 (Development Direction of Private Education). Financial support was gratefully acknowledged. All errors were the sole responsibility of the authors. The authors wished to acknowledge assistance and encouragement from colleagues of the department of civil and construction engineering.

\section{References}

1. Li, Z., Li, G.P. (2009) Empirical research progress on spatial structure models of single center cities. Regional Research and Development. Commun., 28: 5-8.

2. Ding, C.R. (2005) The economic dynamic mechanism of urban pie space expansion. Urban Planning. Commun., 29: 56-60.

3. Alonso, W. (1964) Location and land use. Harvard University Press, Cambridge MA.

4. Mills, E.S. (1967) An aggregative model of resource allocation in a metropolitan area. American Economic Review. Commun., 57: 197-210.

5. Muth,R.F. (1969) Cities and housing. University of Chicago Press, Chicago IL.

6. Wheaton, W.C. (1974) A comparative static analysis of urban spatial structure. Journal of Economic
Theory. Commun., 9: 223-237.

7. Oates, W.E., Schwab, R.M. (1997) The impact of urban land taxation: the pittsburgh experience. National Tax Journal. Commun., 50: $1-21$.

8. Brueckner, J.K, Fansler, D.A. (1983) The economics of urban sprawl: theory and evidence on the spatial sizes of cities. Review of economics and statistics. Commun., 65: 479-482.

9. Alig, R.J., Healy, R.G. (1987) Urban and built-up land area changes in the United States: an empirical investigation of determinants. Land Economics. Commun., 63: 215-226.

10. McGrath, D.T. (2005) More evidence on the spatial scale of cities. Journal of Urban Economics. Commun., 58: 1-10.

11. Song, Y., Zenou, Y. (2006) Property tax and urban sprawl: theory and implications for US cities. Journal of Urban Economics. Commun., 60: 519-534.

12. Lu, L.C. (2006) Suburbanization mechanism of Chinese metropolis in the new economic era: comparison with American suburbanization. Regional research and development. Commun., 25: 6-10. 